\title{
Successful Completion of Upper Gastrointestinal Endoscopy: A Retrospective Comparative Study on Patients who had Endoscopy with Sedation and Without Sedation
}

\author{
Joseph Benjamin Davies', Sunil Kanti Roy ${ }^{2}$ \\ 'William Harvey Hospital, Ashford, Kent, and ${ }^{2}$ Crook Log Surgery, Bexleyheath, London, United Kingdom.
}

\begin{abstract}
Introduction: Sedation of patients before esophagoduodenoscopy (EGD) is common practice. It is expensive, increases the time required to perform the procedure and increases risks of complications. We have set out to determine if sedation improves EGD completion rates.

Methods: 1539 EGDs were performed in the UK by an experienced endoscopist. The age, sex, premedication and completion data made at each procedure were recorded. A completed procedure was defined by the ability of the endoscopist to reach the second part of the duodenum. We measured the difference in the completion rates of EGD between sedated and non sedated patients.

Results: The completion rate for all procedures was $96.6 \% .97 .8 \%$ of procedures were complete in non sedated patients vs. $94.7 \%$ in sedated patients $(p=0.001)$. Male patients were more likely to have the EGD without sedation, as were those over the age of 65. There were no significant differences in the completion rates of EGD between patients who received IV sedation plus topical local anesthesia and those who received IV sedation alone.

Conclusion: The effect of sedation on EGD completion rates is previously undocumented. In order to reduce numbers of failed procedures and prevent exposing patients to the risks of sedation, we suggest these patients should be targeted for more intense pre-procedure counseling. Adequate explanation and reassurance may reduce numbers of patients who request IV sedation by reducing anxiety and increasing expectations of comfort. Reducing numbers of sedated patients may also result in a faster, more cost effective service. (J Dig Endosc 2013;4(2):33-38)
\end{abstract}

Key Words: Upper gastrointestinal endoscopy - Sedation - Completion rate - Patient safety

\section{Introduction}

EGD is a safe and well tolerated procedure that is often being performed with the use of intravenous (IV) sedation, usually with benzodiazepines. ${ }^{2,3}$ The aim of sedation is to increase patient tolerance, reduce anxiety and enable the endoscopist to make an adequate clinical assessment. Currently using IV bendodiazepines to sedate patients prior to EGD is considered reasonably safe in all age groups and the practice is routine due to the perceived unpleasant nature of the procedure.
There is little published data looking at the number and age of patients receiving IV sedation. However, some studies have shown that younger patients have worse pre-procedure expectations and more anxiety compared to older patients. ${ }^{4}$ Anxiety is likely to lead to a request for some form of sedation, which due to widespread and routine use is usually

Reprints requests and correspondence:

Dr. Joseph Benjamin Davies

5 Kippington Drive, Mottingham, London, SE9 4RG

Phone: 07989148889

Email: joseph.davies@doctors.org.uk 
given. In addition, these pre-procedure perceptions have been shown to differ significantly from post procedure reports of discomfort, suggesting that patients may be unnecessarily requesting sedation. ${ }^{4}$

Benzodiazpines enhance the actions of GABA-A resulting in sedation, hypnotic, anxiolytic, anti-convulsant and muscle relaxant effects. Overdose can lead to respiratory depression and hypoxia and the elderly are particularly sensitive to these effects. ${ }^{5,6}$ In response to these risks it is now necessary to use constant patient monitoring such as pulse oximetry, maintain continuous intravenous access and to have equipment available to deal with events such as significant hypoxia and cardiorespiratory arrest. ${ }^{7}$ The safety of IV sedation in the endoscopy suite has long been a topic of debate. Using these drugs without the presence of a trained anaesthetist and a lack of standardized sedation training across the speciality has resulted in some groups calling for restrictions to lower patient exposure to a potentially hazardous event. ${ }^{8}$

Various reports have demonstrated that unnecessarily high doses of Benzodiadepines are often given to frail or elderly patients. ${ }^{9}$ This is despite guidelines recommending lower doses in this group, ${ }^{7,10}$ evidence that older patients tolerate the procedure better and that they are likely to be less anxious prior to the procedure. ${ }^{4}$ Excessive doses of benzodiazepines have been implicated in EGD related mortality rates of 1 in $2000{ }^{11}$ and a NCEPOD report into endoscopic practice in the UK found that sedation was inappropriate in $14 \%$ of post EGD deaths. ${ }^{12}$ Considerable morbidity rates of near $7 \%$ are also associated with sedation. The majority are caused by oversedation in elderly patients . ${ }^{11}$

From the endoscopists perspective, consensus is that a more relaxed and calm patient may be more compliant, making the procedure easier to complete. ${ }^{13}$ In this work we investigate the practice of a single experienced endoscopist to determine the number, age and sex of patients receiving sedation and the association between pre-sedation and completion rates. Our work is aimed towards reducing numbers of incomplete procedures. Patients who had failed EGD and also received sedation, those patients did not have satisfactory clinical outcome of EGD, to the contrary they were also put to the extra risk of side effects of sedation.

\section{Methods}

\section{Study Population}

We performed a retrospective study of the association between intravenous sedation with midazolam and procedure completion rates in patients undergoing EGD in the UK between 1998 and 2011. Data from 1631 randomly selected procedures was analysed. These procedures were carried out by the same experienced endoscopist in two District General Hospitals in the South East of England. These facilities provide elective upper GI endoscopy services to their local populations and carry out in excess of 3000 procedures per year. The endoscopist performs approxi- mately 500 diagnostic endoscopies per year. The mean age of those studied was 55.9 years (range 16 to 95 years), 56.5\% were aged between 35 and 65 at the time of endoscopy and $49.3 \%$ were male.

Study population was taken from a common hospital endoscopy list. It included all diagnostic upper GI endoscopies carried out by this endoscopist between 12/12/1998 and $11 / 2 / 2011$. Raw Data included clinical information organised into 10 fields, recorded retrospectively from endoscopy reports and analysed using Microsoft Excel. Data were anonomysed for the purpose of confidentiality in accordance with the Caldicott Principles. ${ }^{14}$

\section{Inclusion Criteria}

A total of 1631 upper GI endoscopies were carried out of which 1539 met the study inclusion criteria. Inclusion was dependent on complete data fields relating to sex, date of birth, date of procedure, pre procedure medication and completion data (Table 1). Forty two patients were excluded for incomplete DOB, procedure date and gender information and 50 with incomplete premedication fields.

\section{Analysis}

Table 1: Inclusion criteria

Patient data:

Hospital where procedure performed Anonymised unique patient identifier Date of birth

Date of procedure

Sex

Age at time of procedure

Outcome data:

Pre-procedure medication

\section{Completion / failure \\ Endoscopic findings 1 \\ Endoscopic findings 2}

Inclusion in the study was dependent on having each of the above data fields completed for a single Upper Gl endoscopy.

The main objective of the study was to measure procedure completion rate, defined as the ability of the endoscopist to reach the second part of the duodenum and to make any assessment and biopsies necessary to come to a clinical conclusion. Prior to the procedure the endoscopist would discuss with the patient the risks and benefits of undergoing the procedure with or without sedation with intravenous Midazolam. Based on this discussion the patient would make an informed decision and choose whether they wished to be sedated. Sedation rates were calculated for the study population as a whole, for those who received intravenous sedation (both with and without topical local anesthesia) and for those who did not receive intravenous sedation. The completion rates were further analysed to see whether there were any difference because of their age and sex. We compared completion rates in patients above and below 65 years of age. We also compared the proportion of male and female patients in sedated and non sedated groups. 
A sample of both sedated and non sedated patients were surveyed after the procedure to determine their satisfaction with the procedure. Satisfaction was scored from 1 (very unsatisfied) to 5 (very satisfied).

According to the null hypotheses, there was no difference in completion rates, sex ratio or age distribution between sedated and non sedated groups. This was tested using Microsoft Excel. $p$ values were calculated for continuous data from $\mathrm{Z}$, calculated using the standard error of the means. $p<0.05$ (two-tailed) was considered significant. Chi squared values were derived from fourfold tables to calculate $p$ values for binary data, assuming $\mathrm{Z}$ was normally distributed.

\section{Results}

A total of 1631 upper GI endoscopies were performed by a single experienced endoscopist in two District General hospitals in the South East of England between 1998 and 2011, of which 1539 met the study inclusion criteria (Table 1). There was a high incidence of hiatus hernia (64.1\%), gastritis (49.4\%) and esophagitis (21.1\%) among the population studied. A total of 1486 (96.6\%) upper GI endoscopies were recorded as being "complete" and 603 (39.2\%) were performed under intravenous sedation (Tables 2 and 3). Sedated patients were further subdivided into those

Table 2: Procedure completion rates, gender ratios and age distributions for the population studied and for procedures performed with sedation and local anaesthetic and sedation alone

$\begin{array}{cccl}\text { Sample } & \text { Sedated } & \text { Sedation } & p \\ \text { Population } & \text { \& Local } & \text { only } & \text { value }\end{array}$

$\begin{array}{lcccc}\begin{array}{c}\text { Total procedures } \\ \text { performed }\end{array} & 1631 & & & \\ \begin{array}{c}\text { N included in } \\ \text { study (\%) }\end{array} & 1539 & 297(19.3) & 306(19.9) & \\ \begin{array}{c}\text { Complete } \\ \quad \text { Procedures (\%) }\end{array} & 1486(96.6) & 282(94.9) & 290(94.8) & 0.646 \\ \text { Male (\%) } & 761(49.3) & 107(36.0) & 125(40.8) & 0.236 \\ \text { Female (\%) } & 785(50.7) & 190(64.0) & 183(59.2) & 0.236 \\ \text { Age 35 to 65 (\%) } & 872(56.5) & 181(60.9) & 190(62.1) & 0.876 \\ \text { Age under 65 } & 1049(68.0) & 221(74.4) & 216(70.6) & 0.308 \\ \text { Age under 75 } & 1355(87.9) & 269(90.6) & 275(89.9) & 0.581\end{array}$

${ }^{*} p$ values relate to sedation \& local vs. sedation only groups.

receiving sedation in addition to topical local anesthetic and those receiving intravenous sedation alone. No significant differences in completion rate, gender ratio or age distribution were seen between these two groups (Table 2).

Significant differences were found between procedures conducted with IV sedation and those conducted with no IV sedation and it was associated with lower age and female gender (Figure 1). Higher (94.7\% vs 97.8\%, $p=0.001$ ) completion rates were achieved in the non sedated group (Table 3). There were more male patients in the non sedated group (56.5\%) in comparison to the sedated group $(38.5 \%, p<0.001)$ (Table 3$)$. In patients under the age of $65,41.7 \%$ required IV sedation. This contrasts with patients over the age of 65 , where only $33.9 \%$ required sedation $(p=0.014)$ (Table 4$)$. Sedation was associated with lower completion rates in patients over 65 and under 65 in both male and female groups however this association with lower completion rates was statistically significant only in females under 65 (Figure 2).

Most EGD's were performed in patients aged between 60 and $69(n=334)$ (Table 5) and more procedures were carried out without sedation in all age groups except between the ages of 10 and 19 (Figure 3). Patients in the age groups 40

Table 3: Procedure completion rates, gender ratios and age distributions for the population studied

\begin{tabular}{lcccl} 
& $\begin{array}{c}\text { Sample } \\
\text { Population }\end{array}$ & Sedated & $\begin{array}{c}\text { Non } \\
\text { Sedated }\end{array}$ & $\begin{array}{l}p \\
\text { value }\end{array}$ \\
$\begin{array}{l}\text { Total procedures } \\
\text { performed }\end{array}$ & 1631 & & & \\
$\begin{array}{l}\text { N included in study } \\
\text { Complete }\end{array}$ & 1539 & $603(39.2)$ & $936(60.8)$ & \\
$\quad$ procedures (\%) & $1486(96.6)$ & $571(94.7)$ & $915(97.8)$ & 0.001 \\
Male (\%) & $761(49.4)$ & $232(38.5)$ & $529(56.5)$ & $<0.001$ \\
Female (\%) & $778(50.6)$ & $371(61.5)$ & $407(43.5)$ & $<0.001$ \\
Age 35 to 65 (\%) & $872(56.7)$ & $371(61.5)$ & $501(53.5)$ & 0.002 \\
Age under 65 (\%) & $1049(68.2)$ & $437(72.5)$ & $612(65.4)$ & 0.004 \\
Age under 75 (\%) & $1355(88.0)$ & $543(90.0)$ & $812(86.8)$ & 0.034 \\
\hline
\end{tabular}

* $p$ values relate to sedated vs. non-sedated groups.

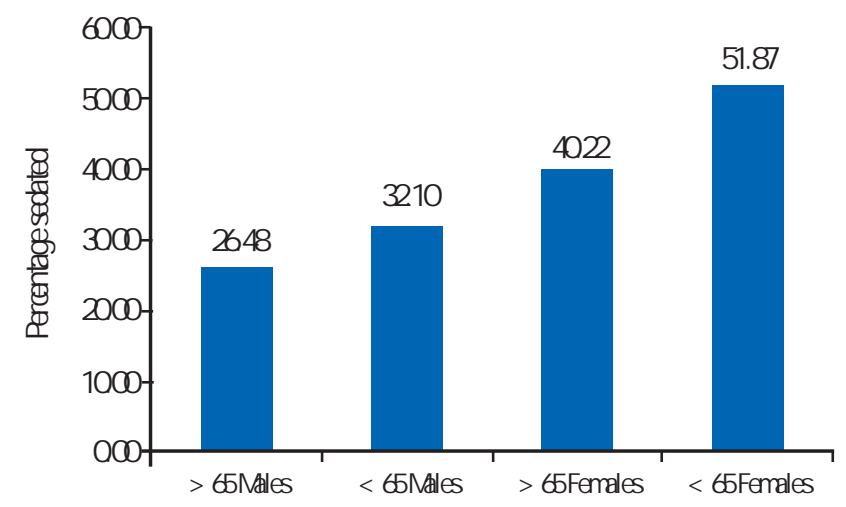

Figure 1: Proportion of patients receiving sedation stratified by sex and age

Table 4: A comparison of the rates of IV sedation in patients aged under 65 with those aged over 65

\begin{tabular}{lccc} 
& Sample Population & Under 65 & Over 65 \\
N 1539 & 1049 & 49 & 0 \\
N Sedated (\%) & $603(39.2)$ & $437(41.7)$ & $166(33.9)$ \\
N Non Sedated (\%) & $936(60.8)$ & $612(58.3)$ & $324(66.1)$ \\
\hline
\end{tabular}

$p$ value for this 2 by 2 table is 0.0144 


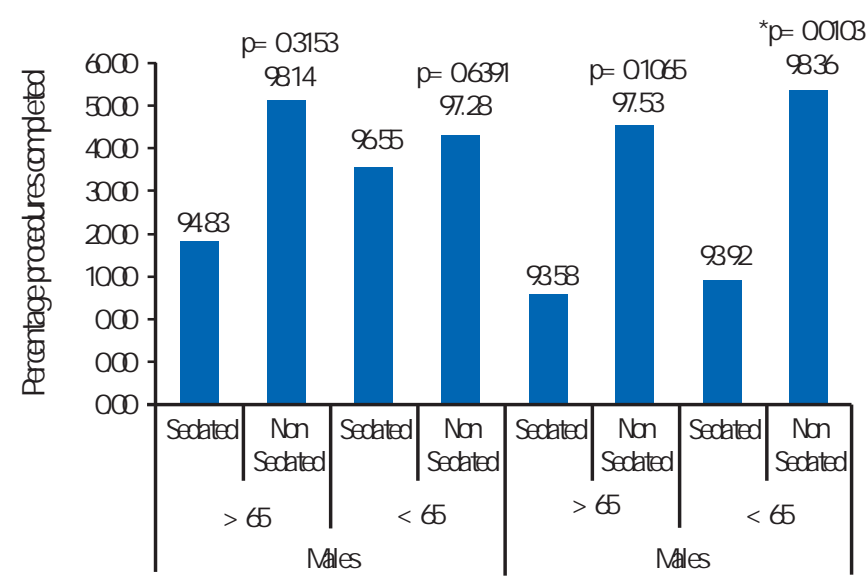

Figure 2: Proportion of completed procedures displayed by sex, age and sedation status.

$p$ values relate to comparison between sedated and non-sedated groups for each age and sex group.

*Denotes statistical significance

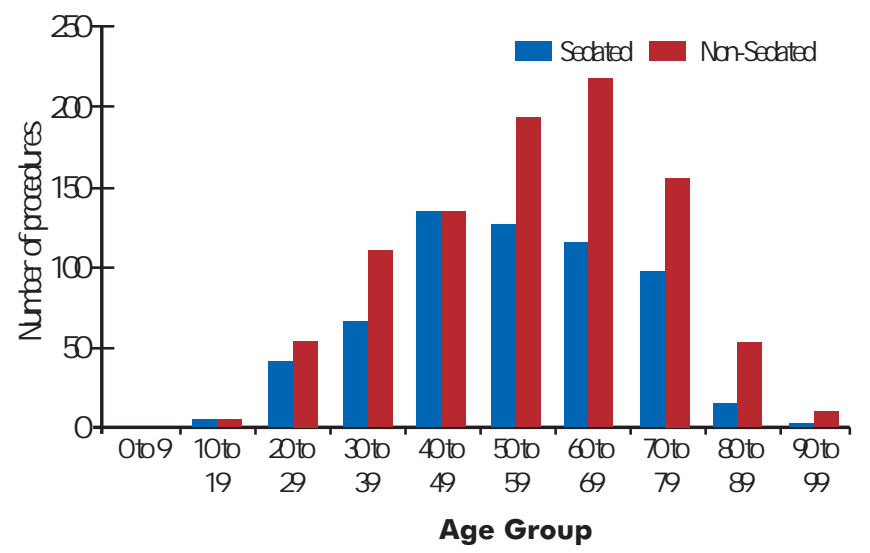

Figure 3: Number of procedures by age group

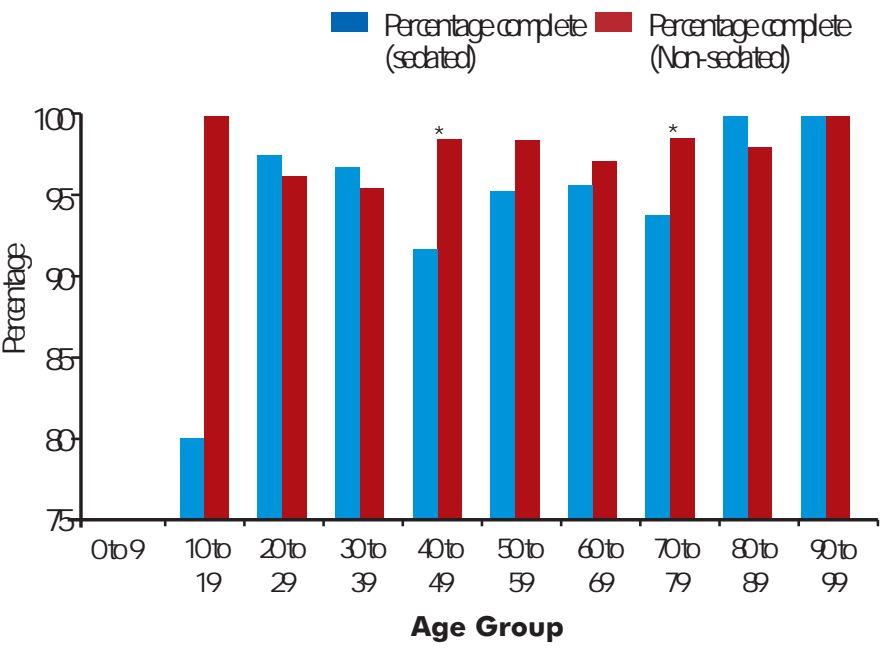

Figure 4: Percentage of completed procedures by age group * Denotes statistical significance Patients in the age groups 40 to 49 and 70 to 79 who were not sedated were significantly more likely to have a complete procedure $(p=0.010$ and $p=0.029$ respectively).

to 49 and 70 to 79 who were not sedated were significantly more likely to have a complete procedure $(p=0.010$ and $p=0.029$ respectively). In no age groups was sedation associated with significantly greater completion rates (Figure 4). Despite sedation being significantly negatively correlated with procedure completion in patients aged between 40 to 49 (Table 5), this group were significantly more likely to be sedated prior to the procedure (Figure 5). More females received sedation than males in both the under 65 and over 65 age groups. In both male and female groups older age was associated with lower sedation levels.

Patient satisfaction was scored 4 or more out of 5 or higher in all patients surveyed with only $1 \%$ of those surveyed reporting dissatisfaction ( 2 or less out of 5 ) with the procedure. There were no significant differences in patient

Table 5: Procedure completion rates by age subgroup

\begin{tabular}{|c|c|c|c|c|c|c|c|}
\hline \multirow[t]{2}{*}{ Age } & \multicolumn{2}{|c|}{ Sample population } & \multicolumn{2}{|c|}{ Sedated } & \multicolumn{2}{|c|}{ Non sedated } & \multirow[b]{2}{*}{$p$} \\
\hline & $\mathrm{N}$ & \% Complete & N & \% Complete & $N$ & \% Complete & \\
\hline 0 to 9 & 0 & N/A & 0 & N/A & 0 & N/A & NA \\
\hline 10 to 19 & 98 & 8.9 & 5 & 80.04 & 100.0 & 0.3711 & \\
\hline 20 to 29 & 95 & 96.8 & 41 & 97.6 & 54 & 96.3 & 0.7284 \\
\hline 30 to 39 & 177 & 96.0 & 66 & 97.0 & 111 & 95.5 & 0.6275 \\
\hline 40 to 49 & 272 & 95.2 & 136 & 91.9 & 137 & 98.5 & 0.0103 \\
\hline 50 to 59 & 322 & 97.2 & 128 & 95.3 & 194 & 98.5 & 0.0973 \\
\hline 60 to 69 & 334 & 96.7 & 116 & 95.7 & 218 & 97.2 & 0.4482 \\
\hline 70 to 79 & 253 & 96.8 & 97 & 93.8 & 159 & 98.7 & 0.0292 \\
\hline 80 to 89 & 67 & 98.5 & 14 & 100.0 & 53 & 98.1 & 0.6073 \\
\hline 90 to 99 & 10 & 100.0 & 1 & 100.0 & 9 & 100.0 & NA \\
\hline
\end{tabular}

* $p$ values relate to sedated vs non-sedated groups 


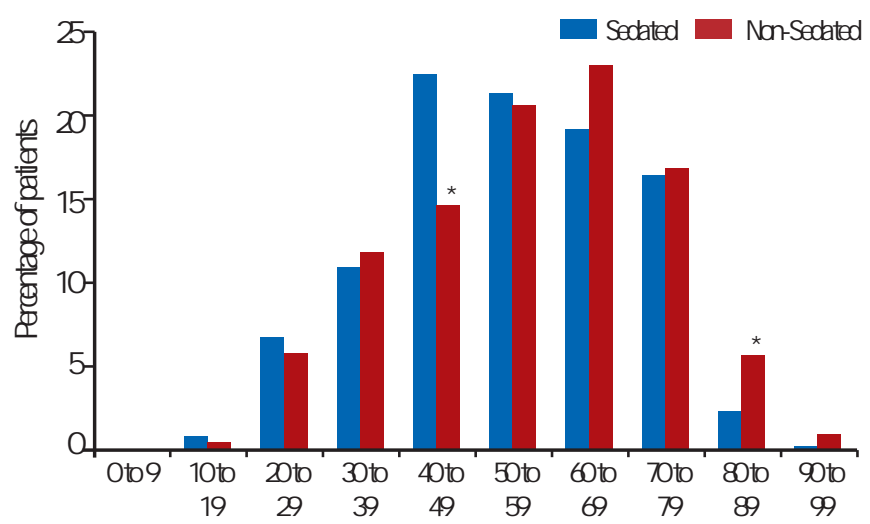

Figure 5: Percentage population in sedated and non-sedated groups by age group

* Denotes statistical significance

satisfaction between those who were sedated and those who did not receive sedation.

\section{Discussion}

Our results show that there are more failed procedures in patients who are sedated. From our experience, this is a result of poor patient tolerance and a reduced ability to comply with instructions during the procedure and not as a result of anatomical abnormalities complicating the procedure. There are likely to be a variety of reasons for the association between IV sedation and higher rates of procedure failure. Primarily, sedation is likely to be a marker of patient anxiety and therefore predicts poor patient tolerance of the procedure. In addition, it is our experience that sedated patients are less able to comply with instructions during the procedure and therefore are unable to assist the endoscopist by positioning themselves in ways to facilitate the passing of the endoscope.

Sedation rates vary significantly between age groups and genders. Females and those aged between 40 and 49 were most likely to be given IV sedation whereas older patients were more likely have the procedure without sedation. Older patients may have had EGD in the past, have lower anxiety levels and there may also be a reluctance to offer IV sedation to this cohort due to increased risks. ${ }^{5,6}$ In both younger and older age groups sedation was associated with lower completion rates and significantly so between the ages of 40 to 49 and 70 to 79 (Chart 4). These results suggest that the morbidity and mortality associated with sedation may not be justified due to the lower completion rates observed in these groups.

Some groups are likely to find unsedated EGD difficult to tolerate and include those undergoing EGD for the first time, patients under the age of 75 and those with high levels of pre procedural anxiety. ${ }^{15}$ Our results compliment these data and show that more young and female patients have sedation. It is likely that sedation is not the only cause for lower completion rates, but it may be a marker for a more anxious and less cooperative patients. However, giving sedation to reduce stress and anxiety did not result in completion rates equal to those in non-sedated patients. The reasons for this are beyond the scope of our data however may relate, in part, to the sedation itself.

In order to reduce numbers of failed procedures and prevent exposing patients to the risks of sedation, which we have shown, may also include procedure failure, we suggest these patients should be targeted for more intense preprocedure counselling. Adequate explanation and reassurance may reduce numbers of patients who request IV sedation by reducing anxiety and increasing expectations of comfort. Reducing numbers of sedated patients may also result in a faster, more cost effective service.

We must accept that this study is limited and it is biased by the fact that a single endoscopist carried out all procedures and we cannot therefore extrapolate these sedation rates to all practitioners. However, these data do reveal useful information relating to the effects of sedation on procedure completion rates. This should be considered when discussing the procedure with the patient and should encourage both the patient and the endoscopist to avoid sedation if possible. We do not have data relating to doses of sedation administered or to rates of complications secondary to the procedure and so we cannot comment on the association between depth of sedation and procedure outcome.

\section{Conclusion}

Through offering patients a thorough and reassuring explanation of the procedure and then of the risks and benefits of sedation, it is the view of these authors that patients may be encouraged to have the procedure without sedation. This may reduce the significant risk of morbidity and mortality and also an increased risk of procedure failure. Lower sedation levels will also result in improvements in efficiency, by reducing time taken to perform each procedure and reduce the level of post procedure monitoring required while recovery takes place. Further research is required to confirm the association between sedation and procedure failure.

\section{References}

1. Al-Atrakchi HA. Upper gastrointestinal endoscopy without sedation: a prospective study of 2000 examinations. Gastrointest Endosc 1989;35:79-81.

2. Daneshmend TK, Bell GD, Logan RFA. Sedation for upper gastrointestinal endoscopy: results of a nationwide survey. Gut 1991;32:12-15.

3. Arrowsmith JB, Gerstman BB, Fleischer DE, Benjamin SB. Results from the American Society for Gastrointestinal Endoscopy/US Food and Drug Administration collaborative study on complication rates and drug use during gastrointestinal endoscopy. Gastrointest Endosc 1991;37:421-27.

4. Thanvi B, Munshi S, Vijayakumar N, Taub N, Lo T. Acceptability of oesophagogastroduodenoscopy without intravenous sedation: patients' versus endoscopist's 
perception with special reference to older patients. Postgrad Med J 2003;79: 650-51.

5. Report from the Boston Collaborative Drug Surveillance Program, Boston University Medical Centre. Clinical depression of the central nervous system due to diazepam and chlordiazepoxide in relation to cigarette smoking and age. $N$ EngJMed 1973;288: 277-80.

6. Greenblatt DJ, Allen MD. Toxicity of nitrazepam in the elderly: a report from the boston Collaborative Drug Surveilance Program. BrJ Clin Pharmac 1978;5:407-13.

7. The British Society of Gastroenterology (BSG) guidelines on sedation, safety and monitoring. November 2003. [Online September 2012].

8. Implementing and ensuring Safe Sedation Practice for Healthcare Procedures in adults. [Online September 2012]. 2001 Report of a Working Party established by the Royal College of Anaesthetists.

9. Lord DA, Bell GD, Gray A, Quine A, Bowles J, Romaya C et al. Sedation for Gastrointestinal Endoscopic Procedures in the Elderly: Getting Safer but Still Not Nearly Safe Enough. [Online September 2012]. 2005; British Society of Gastroenterology.
10. Bell GD, McCloy RF, Charlton JE, Campbell D, Dent NA, Gear MWL, et al. Recommendations for standards of sedation and patient monitoring during GI endoscopy. Gut 1991;32: 823-27.

11. Quine MA, Bell GD, McCloy RF, Charlton JE, Devlin HB, Hopkins A. Prospective audit of upper gastrointestinal endoscopy in two regions of England: safety, staffing and sedation methods. Gut 1995;36:462-67.

12. Cullinane M, Gray AJG, Hargraves CMK, Lucas S, Schubert M, Sherry KM. Scoping our Practice. [Online September 2012]. 2004; NCEPOD.

13. Ross WA. Premedication for upper gastrointestinal endoscopy. Gastrointest Endosc 1989;35:120-26.

14. Caldicott Committee. Report on the review of patient identifiable information. [Online September 2012].1997; Department of Health.

15. Campo R, Brullet E, Monteserrat A, Calvet X, Rivero E, Brontos C. Topical pharyngeal anaesthesia improves tolerance of upper gastrointestinal endoscopy: a randomised double-blind study. Endoscopy 1995;27:659-64.

Source of support: Nil; Conflict of interest: none declared 